\title{
From faceted vesicles to liquid icoshedra: Where topology and crystallography meet
}

\author{
Shani Guttman ${ }^{\mathrm{a}}$, Benjamin M. Ocko ${ }^{\mathrm{b}}$, Moshe Deutsch $^{\mathrm{a}}$, Eli Sloutskin ${ }^{\mathrm{a}, *}$ \\ ${ }^{a}$ Physics Department and Institute of Nanotechnology 83 Advanced Materials, Bar-Ilan University, Ramat-Gan 5290002, \\ Israel \\ ${ }^{b}$ Condensed Matter Physics \&3 Materials Sciences, Brookhaven National Laboratory, Upton, NY 11973, USA
}

\begin{abstract}
Many common amphiphiles spontaneously self-assemble in aqueous solutions, forming membranes and unilamellar vesicles. While the vesicular membranes are bilayers, with the hydrophilic moieties exposed to the solution, the structure formed by amphiphiles at the oil-water (i.e., alkane-water) interfaces, such as the surface of an oil droplet in water, is typically a monolayer. It has recently been demonstrated that these monolayers and bilayers may crystallize on cooling, with the thermodynamic conditions for this transition set by the geometry of the constituent molecules. While a planar hexagonal packing motif is particularly abundant in these crystals, an hexagonal lattice is incompatible with a closed-surface topology, such as a closed vesicle or the surface of a droplet. Thus, (at least) 12 five-fold defects form, giving rise to a complex interplay between the stretching and the bending energies of these two dimensional crystals; in addition, a central role is also played by the interfacial tension. This interplay, part of which has been theoretically studied in the past, gives rise to a range of unexpected and counterintuitive phenomena, such as the recentlyobserved temperature-tunable formation of stable liquid polyhedra, and a tail growing and droplet-splitting akin to the spontaneous emulsification effect.
\end{abstract}

Keywords: emulsion, topological defect, spontaneous emulsification, alkane, surfactant

\section{Inroduction}

Emulsion droplets and surfactant vesicles are both closed-form objects dispersed in a continuous phase. They differ, however, in their structure (the 5 former being a liquid aggregate of molecules while the later - an empty shell-like object), in their thermodynamics (as we discuss below), and in many other properties. Yet, they have one attribute in common: both are stabilized by a layer of surfac10 tants at their interface with the continuous phase. This property renders them capable of assuming closed faceted shapes, on which we focus this brief review.

Surface tension $\gamma$, the excess free energy per unit area of an interface over the bulk[1] is one of the most important properties of a liquid. In particular, since $\gamma>0$, a droplet free from external forces

\footnotetext{
${ }^{*}$ Corresponding author

Email address: eli.sloutskin@biu.ac.il (Eli Sloutskin)
}

will adopt a spherical shape to minimize its surface area $A$ and, thus, its free energy $\gamma A[2]$. The 20 wetting properties of liquids, their mutual miscibility, and wave dynamics at their interfaces, are all determined by $\gamma[1]$. The archetypical immiscible liquids interface, the oil-water one, has a long and distinguished history dating from Hammurabi's 25 codex(18th century BCE) [3], through Aristotle (4th century BCE) and Pliny the Elder (1st century $\mathrm{CE})$, to Benjamin Franklin's celebrated Clapham pond experiment (18th century CE)[4]. Most modern era methods for tuning $\gamma$ of the oil-water inso terfaces employ surfactants. These amphiphilic molecules are admixed to one of the bulk phases and lower $\gamma$ by preferentially adsorbing to the interface.

The simplest amphiphiles, e.g. SDS (sodium 35 dodecyl sulfate), CTAB (cetyltrimethylammonium bromide), long-chain alcohols and fatty acids, consist of a hydrophilic headgroup and a single hydrophobic alkyl tail of length $m \leq 18$. The tails 


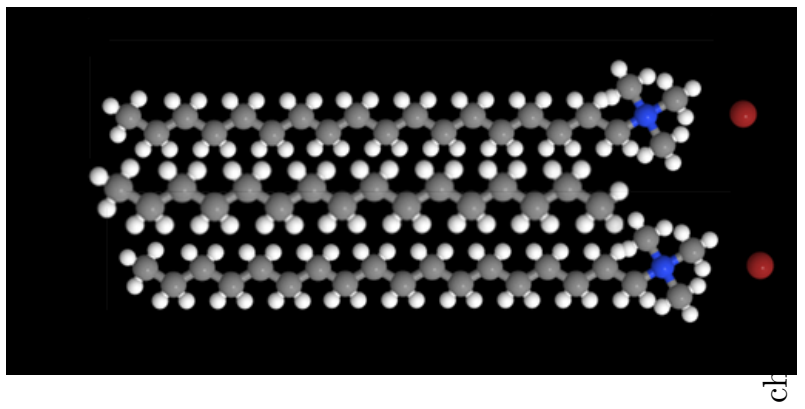

Figure 1: Molecular geometry of an alkane (hexadecane, $\left.\mathrm{H}\left(\mathrm{CH}_{2}\right)_{16} \mathrm{H}\right)$ and of the $\mathrm{C}_{18} \mathrm{TAB}$ surfactant $\left[\mathrm{H}\left(\mathrm{CH}_{2}\right)_{m} \mathrm{~N}(\mathrm{Br})\left(\mathrm{CH}_{3}\right)_{3}, m=18\right]$ allows an efficient packing of a mixture of fully-extended molecules, i.e. cocrystallization. Two surfactants are shown, with an interdigitated alkane. Atom's color code: C - grey, H - white, N - blue, and (dissociated) $\mathrm{Br}^{-}$counterions - red.

are kinked and flexible above the chain melting 40 temperature and linear, fully extended, below it [5]. The equal-charge headgroups Coulomb-repel, while the extended chains van-der-Waals-attract, each other. Thus, the former inhibit, while the latter promote, close packing of the surfactant molecules

45 in the interfacial layer. The balance is usually tipped against attraction-promoted layer crystallization by the steric hinderance of the largercross-section headgroups, which efficiently prevents close contacts between chains. However, the pack50 ing efficiency dramatically improves when a second component is present. This so-called 'cocrystallizing agent' may be an oppositely-charged surfactant or even a simple linear oil molecule (alkane, $\left.\mathrm{H}\left(\mathrm{CH}_{2}\right)_{n} \mathrm{H}\right)$ capable of interdigitating between the 55 fully-extended alkyl tails, thus improving the packing efficiency and maximizing the van der Waals interactions[6] (see Fig. 1).

When cocrystallization is possible, the surfactant tends to form various (quasi-) planar structures[1], so where the radius of curvature (if finite) is larger by several orders of magnitude, compared to the molecular dimensions. In a bulk solution, the surfactant and the cocrystallizing agent will typically form a bilayer membrane, which may close on itself 65 forming a vesicle typically of $0.1-10 \mu \mathrm{m}$ in size $[7,8]$. At the alkane-water and air-water interfaces, a mixed alkane-surfactant monolayer forms[2, 9-11]. ${ }_{105}$ These mono- and bi-layers melt when heated to some temperature $T_{s}$. For $T>T_{s}$, the alkyl tails 70 are highly kinked and disordered. For $T<T_{s}$, the alkyl tails are fully extended and form a (quasi-) two-dimensional hexagonal lattice[2, 7-11]

For non-planar interfaces topology steps in: while
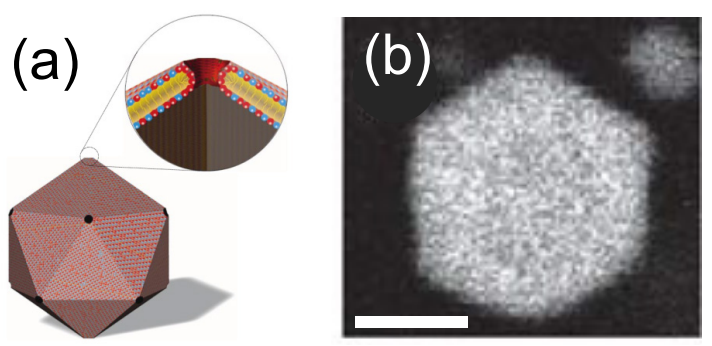

Figure 3: (a) Sketch of an icosahedral vesicle. Reproduced from [7]. (b) Confocal image of a huge icosahedral vesicle. The scale bar is $8 \mu \mathrm{m}$. Reproduced from [25].

hexagons can perfectly tile a flat surface, Euler's 75 formula decrees that at least 12 pentagons must be also included for perfectly tiling the surface of a sphere by hexagons[12-15]. This is why soccer balls have 12 pentagonal patches, in addition to the hexagonal ones. In a crystalline hexagonal lattice tiling a sphere, the 12 five-coordinated sites constitute defects which incur large extensional stresses with associated energy proportional to the total lattice area[12, 16]. The stress is minimized by maximizing the defect separation[15], placing them at the vertices of an inscribed icosahedron, as for 12 electrons freely moving on a spherical surface, in Thomson's model of the atom[15].

The extensional stress can be relaxed by defect buckling, which reduces significantly the local ra9 dius of curvature at the defect location[16], and the energy's area proportionality to a logarithmic dependence. However, buckling also incurs energy penalties for the crystalline layer's bending $[16]$ and for the increase in the surface area imposed by 95 the volume conservation for droplets, though not for vesicles[2]. When these penalties are small, the buckling of the 12 maximally-separated defects may form connecting ridges, and the droplet or the vesicle (Fig. 2(b) and 3, respectively) assume an icosahedral shape. We believe this to be the only known physical mechanism causing a liquid droplet to assume spontaneously a faceted shape, while still remaining liquid (except for a $\sim 2 \mathrm{~nm}$-thick crystallized interfacial monolayer)[2]. We also note that stress relaxation is also possible by the Eulerformula-allowed creation of pairs of 5- and 7-fold defects, which may form string-like grain-boundaries, known as 'scars' [12].

Faceting and other dramatic effects of interface cocrystallization in vesicles and droplets are the focus of this review. Sections 2-4 summarize the 


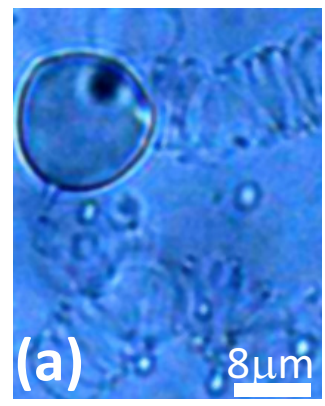

$\mathrm{T}<\mathrm{T}_{\mathrm{SE}}$

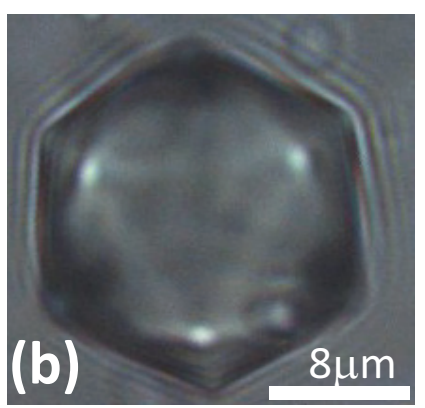

$\mathrm{T}_{\mathrm{SE}}<\mathrm{T}<\mathrm{T}_{\mathrm{d}}$

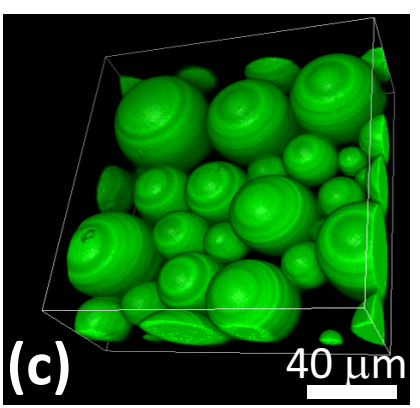

$T>T_{d}$

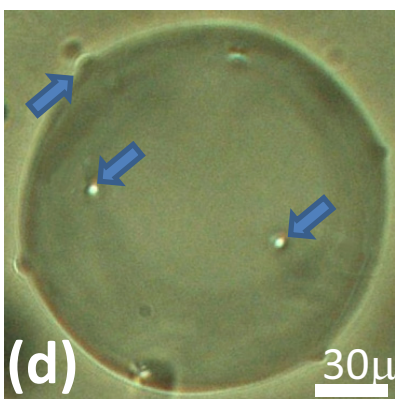

$\mathrm{T}_{\mathrm{SE}}<\mathrm{T}<\mathrm{T}_{\mathrm{d}}$

Figure 2: Optical microscopy images of small (a-c) and large (d) alkane (hexadecane) droplets in an aqueous $\mathrm{C}_{18} \mathrm{TAB}$ solution. At high temperatures, the interfacial tension domination renders the droplets spherical for both crystalline and liquid interfacial monolayer, as long as $\gamma$ is high [see confocal reconstruction in (c)]. The large positive slope of $\gamma$ in the interfacially-frozen regime reduces $\gamma$ dramatically on cooling, so that the interfacially-frozen layer's elasticity dominates the droplets' shape below $T_{d}$. Consequently, the frozen interfacial monolayer buckles, rendering small droplets icosahedral [bright field microscopy image in (b)], while their bulk remains liquid. Note the five ridges emanating from each vertex, a clear signature of an icosahedral symmetry. At even lower temperatures, the interfacial tension becomes transiently-negative, so that the droplets grow tails, which elongate and thin in time [bright field microscopy image in (a)]. Surfactant adsorption from the aqueous solution to the newly created interface decreases its bulk concentration, eventually driving $\gamma \rightarrow 0^{-}$. Large droplets, having significant surface -area $(A)$ and -energy $(\gamma A)$, remain spherical [bright field: (d)], but show protrusions formed by defect buckling [arrows in (d)]. $(\mathrm{b}, \mathrm{d})$ reproduced from $[2]$.

main experimental results on faceting in vesicles, where the cocrystallization is achieved by mix- 140 ing two different surfactants, and in oil-in-water emulsion droplets, where a reversible, temperaturetuned, faceting transition was observed, as well as growth of tail-like protrusions at low temperatures. Section 5 summarizes the different theoretical models and research directions in this field. Finally, in Section 6 we provide some concluding remarks.

It is usual for short reviews as this to be somewhat subjective. Ours is probably not an exception. Length and scope constraints, not lack of impor- ${ }_{15}$ tance, also limited the choice of material included. The present topic impacts on a broad range of fields and subjects, from virology and bacteriology to material engineering and applications. A more complete view of these different fields may be obtained ${ }_{155}$ from the publications listed in the Bibliography.

\section{Faceting of vesicles}

Unilamellar vesicles, empty, closed bilayer mem- 160 branes of surfactant molecules in a solvent, form spontaneously in a wide range of different surfactant solutions[17, 18]. For such vesicles to form, the effective cross-section of the hydrophilic headgroup must be comparable to that of the hydropho- ${ }_{165}$ bic moiety. This is the reason why many doublechained surfactants tend to form vesicles[1, 19]. By cocrystallization of two surfactants, the headgroups of which are oppositely charged in water, the effective combined headgroup cross-section is dramatically reduced, thus allowing an efficient chain packing and a spontaneous assembly of vesicles.

This road to vesicle self-assembly has been documented by Zemb et al. in mixtures of cationic and anionic surfactants, called 'catanionics' $[7,8$, 20]. Employing surfactants with $\mathrm{OH}^{-}$and $\mathrm{H}^{+}$ counterions, salt free, or "true" catanionics[21] are obtained, yielding faceted, icosahedrally-shaped vesicles $[7,8]$. The absence of salt renders the conductivity much lower, and the Debye length much larger, than those due to salt-forming counterions at the same concentration. As the Debye length in this system is unusually large for an aqueous solution $(\sim 100 \mathrm{~nm})$, long-range Coulomb interactions have been first believed to play a central role in determining the shape of these faceted micron-size vesicles $[7,8,20,22]$. However, the observation of faceted vesicles in salt-forming catanionic systems of short Debye lengths[23-25], suggests a different, presently unknown, faceting mechanism[26].

While their Debye lengths vary greatly, all systems exhibiting faceted vesicles comprise long alkyl tails, which must be in their extended, frozen state for faceting to occur[19, 20]. The only exception, where fluid polyhedral vesicles were claimed, included fluorinated tails, where 
amphiphobic interactions may have been complicating the behavior[27]. With extended alkane molecules known to form hexagonally-packed sur- 2 face crystals [5, 9], the in-plane structure of the frozen alkyl tails of catanionic bilayers[20] must also be hexagonal. In a non-planar topology with small bending energy and buckling penalties this should ead to 5-fold defects and their buckling, as dis- 220 cussed above, yielding icosahedral vesicles, with flat facets separated by sharp ridges and vertices[16], as indeed observed $[7,8]$. Furthermore, certain surfactant combinations and impurities, facilitating formation of defect scars, grain boundaries, or in- 22 ducing elastic heterogeneities, may lead to partly faceted shapes instead of perfect icosahedra, as also observed[23, 24, 27-29]. Thus, while a direct confirmation of the buckling mechanism in the bilayer membrane of faceted vesicles is still missing, a qual- 230 itative comparison of all systems where faceted vesicles and droplets[2] occur, strongly suggests that the icosahedral shapes are due to the alkyl tails' packing being hexagonal.

\section{An important note on thermodynamics.}

Before we discuss the faceting phenomena in liquid droplets, a general distinction between the thermodynamics of vesicles and of liquid droplets must be made. In thermodynamic equilibrium, the pop- 245 ulation of vesicles of each size is set by the requirement that the chemical potential in the vesicle is equal to that of the water-dispersed monomers (and of all other surfactant aggregates which may be present in the system). Changing the external 250 parameters of the system, such as the temperature and the concentration of monomers, tweaks the size distribution of the vesicles. However, for a given set of the external parameters, the total summed bulk volume of all vesicles is spontaneously adjusted by 255 the thermodynamics. In contrast, in an emulsion, the droplets' size distribution and the total volume are set by the preparation process. This very important difference between vesicles and emulsion droplets allows for a different set of control knobs 260 for the two systems. In particular, the interfacial tension $\gamma$ plays a central role in the shape determination of liquid droplets, while being irrelevant for the shapes of the vesicles.

\section{Faceting of liquid droplets}

The only system where droplet faceting has hitherto been systematically studied experimentally is an oil-in-water emulsion[2], where the oil is a normal alkane (hexadecane, Fig. 1), and the droplets are stabilized against coalescence by the cationic surfactant $\mathrm{C}_{18} \mathrm{TAB}$ (octadecyltrimethylammonium bromide, Fig. 1). The interfacial layer stabilizing the droplets was first detected at planar alkanewater[10, 11] and air-water[9] interfaces, where, at $T>T_{s}$, it exists as an admixed alkane-surfactant interfacial monolayer consisting of a liquid of kinked and disordered alkanes and surfactants' alkyl tails [Fig.4(a)]. Atomic resolution synchrotron x-ray measurements reveal that upon freezing at $T=T_{s}$ the monolayer becomes a hexagonally-packed crystal, comprising surface-normal, fully-extended alkanes and surfactant tails, and having large, up to mm-size, crystalline coherence lengths [Fig.4(b)]. At the alkane-water interface, this cocrystallized monolayer coexists, unchanged, with the liquid bulk phase of the same alkane[10,11] over the range $T_{f}<T<T_{s}$, where $T_{f}$ is the bulk alkane freezing temperature. This coexistence allows an exchange of alkane molecules between the surface and bulk phases.

The value of $T_{s}$ in these systems is a function of the alkane and surfactant tail lengths, and of the surfactant concentration, $c$. Therefore, a significant control over the droplet faceting phenomena may be achieved, provided that these phenomena occur over the whole range of lengths allowing cocrystallization. Preliminary studies by the present authors suggest that this is indeed the case[2], with similar phenomena occurring not just for different-length homologs of $\mathrm{C}_{18} \mathrm{TAB}$, but also for other alkyl-tail-bearing surfactants, e.g SDS homologs. These observations suggest that the nonequilibrium faceted droplet shapes detected very recently in other alkane-surfactant systems (mostly of incompletely characterized purity) and interpreted as being driven by an incomplete bulk freezing[30], are in fact not frozen but liquid, with the faceting driven by the same mechanism as in our case.

Understanding faceting in emulsion droplets requires an in-depth consideration of the droplet's interfacial tension $\gamma$ in relation with its better-studied flat-interface counterpart. $\gamma$ is defined as the work done to increase the surface by a unit area[1]. To do so in the fixed-volume emulsion droplet entails moving molecules from the bulk to the surface. The 

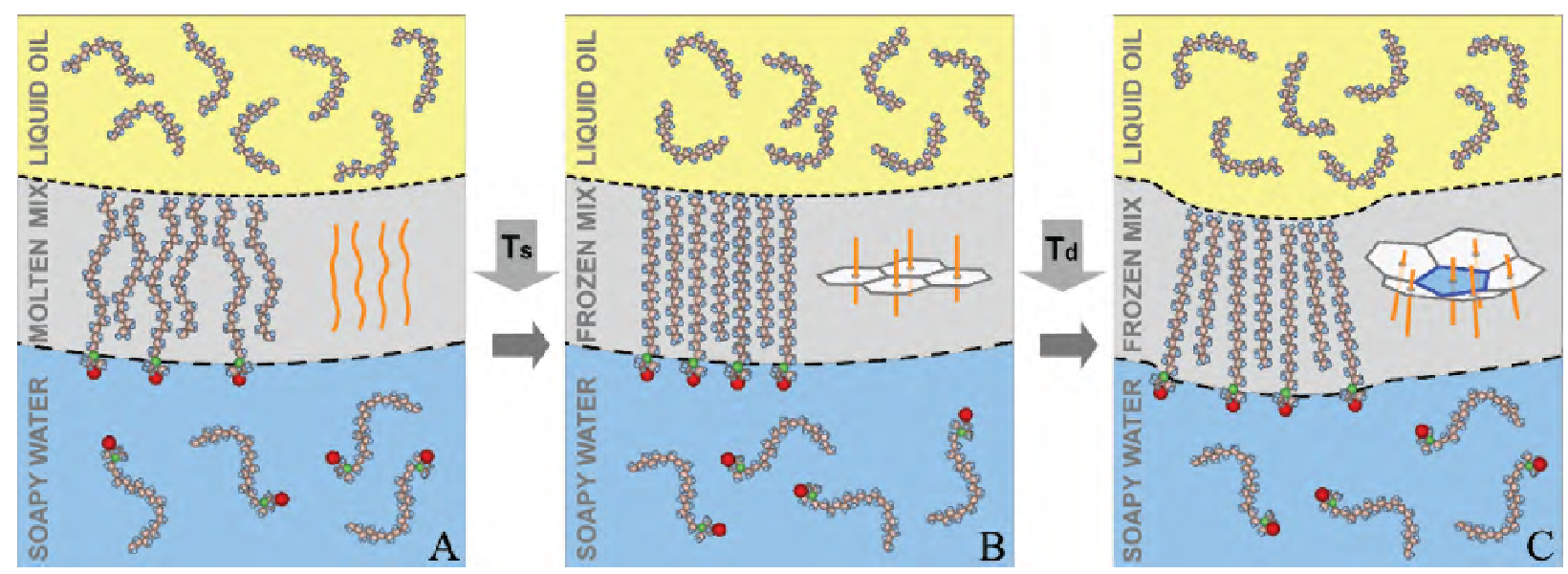

Figure 4: Molecular structure cartoons of the mixed alkane- $\mathrm{C}_{18} \mathrm{TAB}$ interfacial monolayer. (A) Molten interfacial monolayer at $T>T_{s}$. Yellow, blue, green and red denote $\mathrm{C}, \mathrm{H}, \mathrm{N}^{+}$, and $\mathrm{Br}^{-} . \mathrm{C}_{18} \mathrm{TAB}$ headgroups are partially water-ionized and hydrated (not shown). (B) Only the interface freezes at $T=T_{s}$, forming a crystalline, hexagonally-packed, monolayer of extended, interface-normal, molecules[10] (inset). (C) Buckling of a 5-fold defect in an otherwise hexagonally-packed monolayer, residing at the curved interface of an oil droplet in water. Since a closed surface can not be tiled by hexagons, the frozen monolayer includes 12 five-fold defects, associated with a very high in-plane stretching energy. This energy is partly relaxed by buckling, which reduces the local radius of curvature. The inset shows the extended linear conformation of the alkane molecules (orange lines), their hexagonal in-plane packing (as in the B inset), and the buckled defect. Reproduced from [2]. to that of the liquid interface[10,11], as also the 320

entropy loss on bulk crystallization as obtained by calorimetry[5], are both very close to the above estimate of $\Delta S_{i} \approx-k_{B}(n-2) \log 3$. Moreover, a recently developed technique for an in-situ measurement of $\gamma(T)$ in emulsion droplets yields the same $\Delta S_{i}$ as well[2]. These measurements confirm therefore that only a single cocrystallized alkane$\mathrm{C}_{m}$ TAB monolayer forms at the interface: if $N$ layers had been formed, $\Delta S_{i}$ would have been $N$ fold larger[32]. Finally, the fact that the slope stays constant below $T_{s}$ for a broad $T$-range indicates that the monolayer structure is unchanged, in agreement with the x-ray measurements at planar interfaces[10].

The positive $\mathrm{d} \gamma\left(T<T_{s}\right) / \mathrm{d} T$ leads directly to the icosahedral shape change. For high surfactant concentrations approaching the critical micelle concentration (CMC), $T_{s}$ is relatively high and $\gamma\left(T_{s}\right)$ is low (see Fig. 5). Thus, if not preempted by bulk freezing at $T_{f}$, a point may be reached upon cooling where $\gamma(T)$ is low enough for the droplet shape to be dominated by the elasticity of the interfacially-frozen monolayer[2]. Thus, below this $T=T_{d}$ elasticity renders the droplet icosahedral [Fig. 2(b)], with the in-plane stress due to the topological 5-fold defects partially relaxed by their buckling. Above $T_{d}$ droplets are $\gamma$-dominated and spherical [Fig. 2(c)], with the buckling suppressed by the 


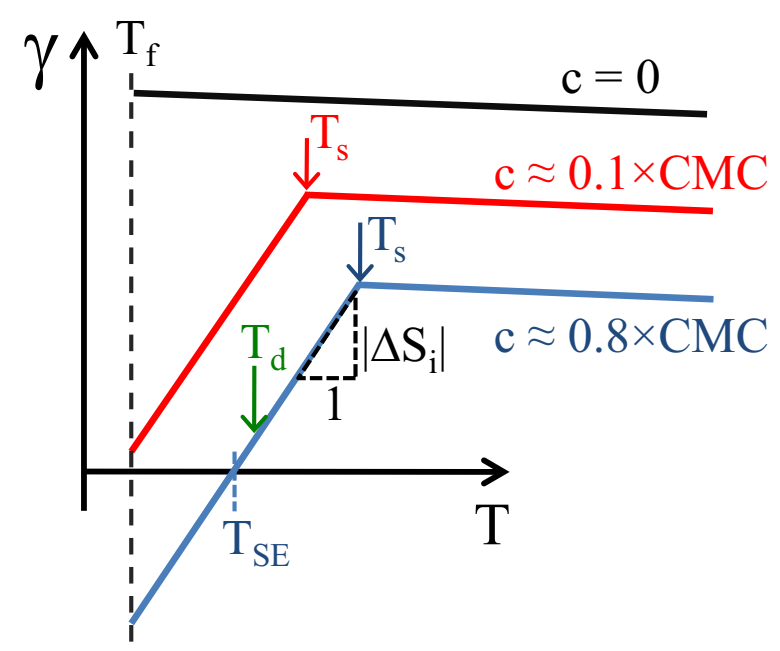

Figure 5: Schematic $\gamma(T)$ of the oil-water interface, at different $\mathrm{C}_{m} \mathrm{TAB}$ surfactant concentrations $c$. The interfacial freezing is observed at $T=T_{s}$ as a sharp slope change in both of the bottom curves. The effect does not occur in the absence of surfactants, i.e. for $c=0$ (upper curve), where the interface remains liquid down to the alkane's bulk freezing at $T_{f}$. The $\gamma(T)$ slope change at $T=T_{s}$ equals the loss in the excess interfacial entropy over the bulk, $\left|\Delta S_{i}\right|$. As $c$ approaches the CMC (critical micelle concentration), $\gamma$ vanishes at $T=T_{S E}$. Near $T_{S E}, \gamma(T)$ is small and the interfacial energy is dominated by the elasticity of the frozen interfacial monolayer, so that the liquid emulsion droplets, initially spherical [see confocal reconstruction in Fig. 2(c)], deform into icosahedral shapes (Fig. 2(b)). Transient negative values of $\gamma$, at $T<T_{S E}$, drive an increase in the interfacial area of the droplets by, e.g., producing nano-coils emanating from a large emulsion droplet in Fig. 2(a)

$\gamma \Delta A$ term of their free energy, where $\Delta A$ is the area difference between the faceted body and a sphere of the same volume. Thus, the droplet's shape can be controlled (reversibly!) by temperature-tuning $\gamma$. Remarkably, the large $\gamma \Delta A$ makes the largest droplets remain spherical, showing just 12 tiny protrusions [Fig. 2(d)], down to $T_{S E}$, where $\gamma$ vanishes. Such control of shapes by $\gamma$ is impossible for vesicles. Tweaking the chemical potential of surfactants inside the vesicular membrane allows the fraction of such surfactants, out of the total surfactant concentration $c$, to be varied; yet, the shape of the vesicles is completely determined by the elasticity of the membrane and its response to an external pressure, if applied[25, 33].

Arguably, the most dramatic phenomena are observed when the situation depicted in Fig. 5c occurs. Since $\mathrm{d} \gamma / \mathrm{d} T>0$ for $T<T_{s}$, for a low enough $\gamma\left(T_{s}\right)$ and high enough $T_{s}$ a critical tem- vanishes, if not preempted by bulk freezing, $T_{f}$. At $T=T_{S E}$ our macro-emulsion is thermodynamically stable, unlike common kinetically-stabilized macroemulsions. We note that similar phenomena, ${ }_{45}$ interpreted in terms of Pickering emulsions, have been previously detected for alkane droplets, stabilized by catanionic surfactant mixtures[34].

As droplets are cooled below $T_{S E}, \Delta \mu$ becomes negative, with the surface entropy loss being overbalanced by the reduction of intrinsic energy in the cocrystallized monolayer. Thus, transiently, $\gamma\left(T_{S E}\right)<0$, leading to spontaneous growth of the interfacial area. In this regime, active dynamics sets in, with the droplets exhibiting spontaneous splitting[2] and growing of tail-like protrusions from their vertices [Fig. 2(a)]. Importantly, while these phenomena have a lot in common with the classical spontaneous emulsification en route to a microemulsion[35], they are fully driven by the interfacial freezing effect. Thus, sharp and faceted[2], rather than fuzzy, diffuse, and budding[36, 37] interfaces occur, limited by the significant elasticity of the interfacially-frozen monolayer. Eventually, the incorporation of the surfactant molecules from the bulk solution into the newly created interface reduces the surfactant's bulk concentration, so that the (negative) $\gamma$ reverts to zero and the thermodynamical equilibrium is reestablished. Moreover, rapid growth of tail-like protrusions may oc, 'mother'-droplet to a spherical shape [Fig. 2(a)], possibly due to formation of surfactant gradients during this rapid dynamics. Although sharp tubular objects have been previously observed in some related systems, it is yet unknown whether the mechanism in these cases is the same as in our case[38, 39].

While the full physical understanding of the taillike tubular protrusions formed in liquid droplets at $T<T_{S E}$, with their cross-sections below the optical resolution [Fig. 2(a)], is still to be established, it is highly tempting to speculate that similar physics may be responsible for the formation of membranous intercellular nanotubes, allowing metabolic cross-feeding among some of the common bacteria[40]. We believe that extensive future studies of simple biomimetic model systems, such as the tail-growing liquid droplets, may potentially allow the fundamental physics of morphogenesis to be revealed, at least at the level of bacteria[41] and their colonies. 


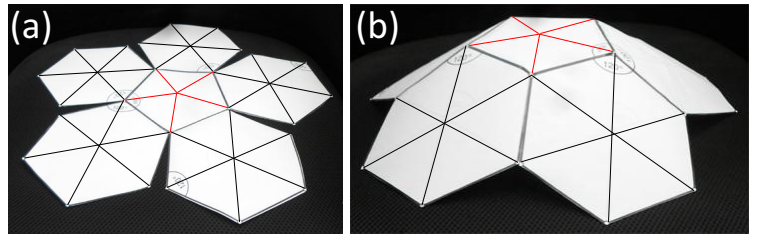

Figure 6: The inextensible limit $\left(\Gamma^{\nu K} \rightarrow \infty\right)$ of a defectcontaining membrane. (a) A pentagonal patch, connected to hexagonal patches of the same edge length. For a planar 435 lattice to be formed, the bonds must stretch, which is impossible at the inextensible limit. Sector-shape mismatches, incurring an energetic cost which grows linearly in the total area of the lattice $\left(R^{2}\right)$, make the membrane buckle into the third dimension $(\mathrm{b})$.

\section{Faceting of closed crystalline membranes: Theoretical models}

Significant theoretical effort focused over the ${ }_{445}$ years on elucidating the phase behavior of twodimensional hexagonal crystals, where topological lattice defects play a central role[42]. While the ground state of planar hexagonal crystals does not include any defects, the situation is different for ${ }_{450}$ closed membranes. Here, a minimum of 12 fivefold defects (known as 'disclinations') is dictated by the topology, so that significant stresses are present even in the ground state[12-15]. Whether these stresses can be relaxed by defect buckling, 455 depends on the Föppl-von Kármán $(\mathrm{FvK})$ number, $\Gamma^{\nu K} \equiv Y_{2 D} R^{2} / \kappa$, where $Y_{2 D}$ is the two-dimensional Young modulus, $\kappa$ is the bending modulus, and $R$ is the radius of the spherical membrane. The limit of $\Gamma^{\nu K} \rightarrow \infty$, where the bending energy is negli- 460 gible and/or stretching is completely prohibited, is emonstrated in Fig. 6, employing a paper model. Here, the mismatch in a planar lattice, due to the presence of a five-fold defect, is fixed by an out-ofplane buckling of the lattice[43]. In a more realistic situation of finite $\Gamma^{\nu K}$, the buckling has been predicted[16] to occur for $\Gamma^{\nu K}=\Gamma_{b}^{\nu K} \approx 150$. For a spherical geometry, where 12 disclinations are dictated by the Euler's theorem, the buckling transition, albeit slightly more rounded, is still taking 470 place[16] at $\Gamma^{\nu K}=\Gamma_{b}^{\nu K}$; interestingly, an almost 420 two-fold larger value of $\Gamma_{b}^{\nu K}$ is quoted in some other studies, for exactly the same model and the same conditions $[44,45]$.

For a full account of the theoretical studies of 475 closed crystalline membranes, beyond the scope of the current paper, the reader is referred to the detailed reviews of this exciting field by Bowick and others $[29,46]$. The main approaches to the modeling of the buckling transition in these systems include: continuum-level analytical elasticity arguments[16, 45, 46], numerical energy minimization of a discretized Hamiltonian[2, 14, 16, 29, 44, 45, 47], or finite-temperature Monte-Carlo simulations of a discretized membrane composed of tethered hard spheres[28]. While the energy minimization is strictly valid only at $T=0 \mathrm{~K}$, it seems that the elastic constants in (at least) some of the relevant experimental systems are sufficiently large to justify this choice[2]. In some computer studies of the discretized model, the defects' positions are fixed ('frozen'), with the triangulation of the membrane preserved; thus, screening of elastic stresses by the creation of pairs of 5 -fold and 7 -fold defects was not allowed $[2,14,16,29,44,45]$. This choice is probably justified for $\Gamma^{\nu K}>\Gamma_{b}^{\nu K}$, where the dominant stress relaxation mechanism is the buckling. Indeed, studies where defect creation and annihilation are allowed $[28,47]$, indicate that the contribution of defect pairs is the largest in the unbuckled regime $\left(\Gamma^{\nu K}<\Gamma_{b}^{\nu K}\right)$. In this regime, the energy is reduced by the additional defects; thus, the $\Gamma_{b}^{\nu K}$ value is slightly larger, at least in the finite-temperature studies[28, 47], when such additional defects are allowed. In some of these works, the volume enclosed by the membrane is preserved through the buckling transition[2, 44, 47], such as in the liquid droplets; other studies allow the enclosed volume to vary[16, 29, 45]. Controlled inflation and deflation of membranes has been studied as well[14, 44]. In general, the buckling transition does not strongly depend on volume conservation[44, 47]. However, the role of the additional defect pairs is more pronounced when the enclosed volume is fixed. To preserve the enclosed volume, the buckled shape is more rounded than a true icosahedron, so that the in-plane lattice stresses are not fully relaxed, giving rise to spontaneous formation of additional defect pairs[47]. Finally, multicomponent membranes with elastic heterogeneities have been studied by energy minimization, with the volume unconstrained and the triangulation preserved. In these works, segregation of the softer component to vertices and edges of the membrane allows partial, rather than complete, faceting to be achieved [29, 48], as observed in some experiments[24, 27]. The effect of a non-linear term in the bending energy[29], as also spontaneous curvature contributions[45], may yield non-icosahedral shapes as well, with the facets being either sharp[29] 
or partially curved[45].

Remarkably, while in the experiments on liquid 530 droplets molecular exchange between the interfacially frozen monolayer and the bulk phase plays an important role, as discussed above, such an exchange has not been taken into account in any of the existing theoretical models. This exchange, with the monolayer phase being subject to grand canonical ensemble conditions, is responsible for surface area minimization in liquid droplets; this term is different from the stretching energy, which actually tends to increase the surface area in the case of the buckling. Only one study considers the energy minimization term[2]. However, this work had the triangulation network preserved, so that the actual incorporation of molecules into the crystalline layer, possibly inducing additional disclinations and dislocations, has not been allowed. In addition, the $Y_{2 D}$ and $\kappa$ values obtained in this work by direct optical tweezing experiments and theory do not conform[2] to the classical thin plate theory $[14,16]$. According to the classical theory, $Y_{2 D} / \kappa=12\left(1-\nu^{2}\right) h^{-2}$, where $h \approx 2 \mathrm{~nm}$ is the thickness of the interfacially-frozen monolayer. The disagreement with the classical $Y_{2 D} / \kappa$ relation suggests that the continuum elasticity, the basis of 550 most buckling models, may become invalid at a scale of individual molecules. Clearly, at least in the case of the liquid droplets, additional theoretical work is called for to settle these issues and to 555 describe the phenomena of faceting and tail growth.

\section{Concluding Remarks}

We have briefly reviewed some of the experimental and theoretical work on buckling in closed two-dimensional crystals. Particular attention was given to the recent experiments, where faceting of 565 liquid droplets has been detected. In this work we mainly focused on systems where buckling occurs due to cocrystallization of molecules bearing single linear alkyl tails. However, several im- 570 portant experimental systems where icosahedral shapes have been observed had to be left out: large viruses[16, 45, 49], carboxysomes[50], and nanoshells[51], to mention but a few[46]. Much of the ${ }^{575}$ theoretical insight into the driving mechanisms of buckling may possibly be extended to some or all of these systems. The interplay between the local order and the topological constraints is a subject of intense research in a wide variety of fields, including, but not limited to, active nematics on a spherical shell[52] and toroidal liquid crystalline objects[53]. These studies not only reveal the beauty of the fundamental geometry of nature, but can also be employed in the future to control spontaneous selfassembly of complex shape objects, allowing new building blocks to be formed for future metamaterials.

\section{Acknowledgments}

We thank T. Zemb, D. C. Rapaport, S. A. Safran and Z. Sapir for discussions. Acknowledgment is made to the Donors of the American Chemical Society Petroleum Research Fund for support of this research. B.M.O acknowledges supported by the U.S. Department of Energy, Office of Science, Office of Basic Energy Sciences, under Contract No. DE-SC0012704.

\section{References}

[1] Israelachvili JN. Intermolecular and surface forces (2nd Ed., Academic Press, 1998).

[2] ** Guttman S, Sapir Z, Schultz M, Butenko AV, Ocko BM, Deutsch M, Sloutskin E, How faceted liquid droplets grow tails. Proc Natl Acad Sci USA 2016;113(3):493-496. Study of faceting and spontaneus emulsification in $\mathrm{C}_{18}$ TAB-stabilized alkane-inwater emulsion droplets, clearly demosntrated to arise from interfacial freezing.

[3] Tabor D, Babylonian lecanomancy: An Ancient text on the spreading of oil on water . J. Coll. Interf. Sci 1980; 75(1):240-245.

[4] Franklin B, Of the stilling of waves by means of oil. Phil. Trans. Res. Soc. London 1774; 64(1): 445-460

[5] Ocko BM, Wu XZ, Sirota EB, Sinha SK, Gang O, Deutsch M. Surface freezing in chain molecules: Normal alkanes. Phys Rev E 1997;55(3):3164-3182.

[6] Maoz R, Cohen H, Sagiv J. Specific nonthermal chemical structural transformation induced by microwaves in a single amphiphilic bilayer self-assembled on silicon. Langmuir 1998;14(21):5988-5993.

[7] ** Dubois M, Demé B, Gulik-Krzywicki T, Dedieu JC, Vautrin C, Désert S, Perez E, Zemb T. Self-assembly of regular hollow icosahedra in salt-free catanionic solutions. Nature 2001;411(6838):672-675. Seminal observation of faceted vesicles.

[8] * Dubouis M, Lizunov V, Meister A, Gulik-Krzywicki T, Verbavatz JM, Perez E, Zimmerberg J, Zemb T. Shape control through molecular segregation in giant surfactant aggregates. Proc Natl Acad Sci USA 2004;101(42):15082-15087. A comprehensive experimental study of catanionic vesicle faceting. The theory presented, assigning the shape to excess charges at edges, was later demonstrated by the same group to be incomplete (Ref. [26]).

[9] * Sloutskin E, Sapir Z, Bain CD, Lei Q, Wilkinson KM, Tamam L, Deutsch M, Ocko BM. Wetting, mixing, and phase transitions in Langmuir-Gibbs films. Phys Rev 
Lett 2007;99(13):136102. First study of interfacial freezing of mixed alkane-surfactant monolayes at the flat 650 water-air interface, which reveals the hexagonal molecular packing and large crystalline coherence lengths of the interface-frozen monolayer.

[10] * Tamam L, Pontoni D, Sapir Z, Yefet S, Sloutskin E, Ocko BM, Reichert H, Deutsch M. Modification of 655 deeply buried hydrophobic interfaces by ionic surfactants. Proc Natl Acad Sci USA 2011; 108(14):55225525. First study of interfacial freezing at a flat interface of bulk alkanes with an aqueous surfactant solution, and determination of the frozen monolayer's structure.

[11] Sloutskin E, Bain CD, Ocko BM, Deutsch M. Surface freezing of chain molecules at the liquid-liquid and liquid-air interfaces. Faraday Discuss 2005;129:339-152.

[12] Bausch AR, Bowick MJ, Cacciuto A, Dinsmore AD, Hsu MF, Nelson DR, Nikolaides MG, Travesset A, 665 Weitz DA. Grain boundary scars and spherical crystallography. Science 2003;299(5613):1716-1718.

[13] Meng G, Paulose J, Nelson DR, Manoharan VN. Elastic instability of a crystal growing on a curved surface. Science 2014;343(6171):634637.

[14] Yong EH, Nelson DR, Mahadevan L. Elastic platonic shells. Phys Rev Lett 2013;111(17):177801.

[15] Bowick M, Cacciuto A, Nelson DR, Travesset A. Crystalline order on a sphere and the generalized Thomson problem. Phys Rev Lett 2002;89(18):185502.

[16] ** Lidmar J, Mirny L, Nelson DR. Virus shapes and buckling transitions in spherical shells. Phys Rev E 2003;68(5):051910. Seminal theoretical study of buckling induced shape changes in closed shells.

[17] Jung HT, Coldren B, Zasadzinski JA, Iampietro DJ, 680 Kaler EW. The origins of stability of spontaneous vesicles. Proc Natl Acad Sci USA 2001;98(4):1353-1357.

[18] Coldren BA, Warriner H, van Zanten R, Zasadzinski JA, Sirota EB. Flexible bilayers with spontaneous curvature lead to lamellar gels and spontaneous vesicles. 685 Proc Natl Acad Sci USA 2006;103(8):2524-2529.

[19] Quemeneur F, Quilliet C, Faivre M, Viallat A, PépinDonat B. Gel phase vesicles buckle into specific shapes. Phys Rev Let 2012;108(10):108303.

[20] Zemb T, Dubois M, Demé B, Gulik-Krzywicki T. Self- 690 assembly of flat nanodisks in salt-free catanionic surfactant solutions. Science 1999;283(5403):816-819.

[21] Hartmann MA, Weinkamer R, Zemb T, Fischer FD, Fratzl P. Switching mechanics with chemistry: a model for the bending stiffness of amphiphilic bilayers with 695 interacting headgroups in crystalline order. Phys Rev Lett 2006;97(1):018106.

[22] Vernizzi G, de la Cruz MO. Faceting ionic shells into icosahedra via electrostatics. Proc Natl Acad Sci 2007;104(47):18382-18386.

[23] Antunes FA, Brito RO, Marques EF, Lindman B, Miguel M. Mechanisms behind the faceting of catanionic vesicles by polycations: chain crystallization and segregation. J Phys Chem B 2007;111(1):116-123.

[24] Greenfield MA, Palmer LC, Vernizzi G, de la 705 Cruz MO, Stupp SI. Buckled membranes in mixedvalence ionic amphiphile vesicles. J Am Chem Soc 2009;131(34):12030-12031.

[25] * Béalle G, Jestin J, Carrière D. Osmotically induced deformation of capsid-like icosahedral vesicles. Soft 710 Matter 2011;7(3):1084-1089. An important observation of clear faceted vesicles in a low-Debye-length system, with a qualitative interpretation in terms of the theory in Ref. [16]

[26] * Michina Y, Carrière D, Charpentier T, Brito R, Marques EF, Douliez JP, Zemb T. Absence of lateral phase segregation in fatty acid-based catanionic mixtures. J Phys Chem B 2010;114(5):1932-1938. Recent experimental reexamination of some of the mechanisms underlying the theory of Ref. [8] are questioned by the same group and seem not to be as central as originally believed.

[27] González-Pérez A, Schmutz M, Waton G, Romero MJ, Krafft MP. Isolated fluid polyhedral vesicles. J Am Chem Soc 2007;129(4):756-757.

[28] ** Kohyama T, Gompper G. Defect scars on flexible surfaces with crystalline order. Phys Rev Lett 2007;98(19):198101. Important study of buckling in closed shells by simulations allowing defect mobility and defect-pair production.

[29] ** Bowick MJ, Sknepnek R. Pathways to faceting of vesicles. Soft Matter 2013(34);9:8088-8095. Recent insightful simulations of buckling in vesicles.

[30] * Denkov N, Tcholakova S, Lesov I, Cholakova D, Smoukov SK. Self-shaping of oil droplets via the formation of intermediate rotator phases upon cooling. Nature 2016;528(7582):392-395. A very recent observation of faceting in surfactant-stabilized emulsion droplets, akin to Ref. [2], but in non-thermodynamic-equillibrium situations, and assigned (erroneously, in our view) to a partial bulk crystallization rather than interfacial freezing.

[31] Guggenheim EA. Mixtures (Oxford, Clarendon Press, 1952).

[32] Sloutskin E, Gang O, Kraack H, Doerr A, Sirota EB, Ocko BM, Deutsch M. Surface freezing in binary mixtures of chain molecules. II. Dry and hydrated alcohol mixtures. Phys Rev E 2003;68(3):031606.

[33] Delorme N, Dubois M, Garnier S, Laschewsky A, Weinkamer R, Zemb T, Fery A. Surface immobilization and mechanical properties of catanionic hollow faceted polyhedrons. J Phys Chem B 2006;110(4):1752-1758.

[34] Schelero N, Stocco A, Möhwald H, Zemb T. Pickering emulsions stabilized by stacked catanionic microcrystals controlled by charge repulsion. Soft Matter 2011;7(22):10694-10700.

[35] Granek R, Ball RC, Cates ME. Dynamics of spontaneous emulsification. J Phys II 1993;3(6):829-849.

[36] Shahidzadeh N, Bonn D, Meunier J. A new mechanism of spontaneous emulsification: Relation to surfactant properties. Europhys Lett 1997;40(4):459464.

[37] Sumino Y, Kitahata H, Seto H, Yoshikawa K. Dynamical blebbing at a droplet interface driven by instability in elastic stress: a novel self-motile system. Soft Matter 2011:7:3204-3212.

[38] Haidara H. Wetting-mediated collective tubulation and pearling in confined vesicular drops of DDAB solutions. Soft Matter 2014;10(47):9460-9469.

[39] Volkmer D, Tugulu S, Fricke M, Nielsen T. Morphosynthesis of star-shaped titania-silica shells. Angew Chem Int Ed 2003;42(1):58-61.

[40] Pande S, Shitut S, Freund L, Westermann M, Bertels F, Colesie C, Bischofs IB, Kost C. Nat Commun 2015;6:6238.

[41] Daum B, Quax TEF, Sachse M, Mills DJ, Reimann J, Yildiz O, Hader S, Saveanu C, Forterre P, Albers SV, Kuhlbrandt W, Prangishvili D. Self-assembly of the general membrane-remodeling protein PVAP into sev- 
enfold virus-associated pyramids. Proc. Natl. Acad. Sci. USA 2014;111(10):3829-3834.

[42] Chaikin PM, Lubensky TC. Principles of condensed matter physics (Cambridge University Press, 1995).

[43] Witten TA, Li H. Asymptotic shape of a fullerene ball. Europhys Lett 1993;23(1):51-55.

[44] Šiber A. Buckling transition in icosahedral shells subjected to volume conservation constraint and pressure: relations to virus materation. Phys Rev E 2006;73(6):061915.

[45] Nguyen TT, Bruinsma RF, Gelbart WM. Elasticity theory and shape transitions of viral shells. Phys Rev E 2005;72(5):051923.

[46] Bowick BJ, Giomi L. Two-dimensional matter: order, curvature and defects. Adv Phys 2009;58(5):449-563.

[47] Funkhouser CM, Sknepnek R, de la Cruz MO. Topological defects in the buckling of elastic membranes. Soft Matter 2013;9(1):60-68.

[48] Vernizzi G, Sknepnek R, de la Cruz MO. Platonic and Archimedean geometries in multicomponent elastic membranes. Proc Natl Acad Sci USA 2011;108(11):4292-4296.

[49] Lošdorfer Božic A, Šiber A, Podgornik R. Statistical analysis of sizes and shapes of virus capsids and their resulting elastic properties. J Biol Phys 2013;39(2):215228.

740 [50] Yeates TO, Tsai Y, Tanaka S, Sawaya MR, Kerfeld CA. Self-assembly in the carboxysome: a viral capsidlike protein shell in bacterial cells. Biochem Soc Trans 2007;35(3):508-511.

[51] Huang Q, Yu D, Xu B, Hu W, Ma Y, Wang Y, Zhao Z, Wen B, He J, Liu Z, Tian Y. Nanotwinned diamond with unprecedented hardness and stability. Nature 2014;510(7504):250-253.

[52] Keber FC, Loiseau E, Sanchez T, DeCamp SJ, Giomi L, Bowick MJ, Marchetti MC, Dogic Z, Bausch AR. Topology and dynamics of active nematic vesicles. Science 2014;345(6201):1135-1139.

[53] Pairam E, Vallamkondu J, Koning V, van Zuiden BC, Ellis PW, Bates MA, Vitelli V, Fernandez-Nieves A. Stable nematic droplets with handles. Proc Natl Acad Sci USA 2013;110(23):9295-9300 